\title{
Using Electroporation to Determine Function of a Chimeric Antigen Receptor in T Cell and Macrophage Cell Lines
}

\author{
Carmen S.M. Yong ${ }^{1, *}$, Connie P.M. Duong ${ }^{1,2}$, Christel Devaud ${ }^{1}$, Liza B. John ${ }^{1}$, Phillip K. Darcy ${ }^{1,3} \wedge$ \\ and Michael H. Kershaw ${ }^{1,3, \wedge}$ \\ ${ }^{1}$ Cancer Immunology Research Program, Sir Peter MacCallum Department of Oncology, University of Melbourne, \\ Parkville, Victoria 3010 Australia \\ ${ }^{2}$ Department of Pathology, University of Melbourne, Parkville, Victoria 3010 Australia \\ ${ }^{3}$ Department of Immunology, Monash University, Prahran Victoria 3181 Australia
}

\begin{abstract}
Chimeric antigen receptors (CARs) have been used extensively in adoptive immunotherapy to modify T lymphocytes (CTLs), conferring the effector abilities of these cells towards a tumor-associated antigen (TAA). However, the ability of a CAR to redirect the effector function in a range of immune cells has not been well characterized, mainly due to the limitations in current genetic modification techniques. To overcome these limitations, we used the rapid technique of electroporation to transiently modify a macrophage, $\mathrm{CD}^{+} \mathrm{T}$ and $\mathrm{CD}^{+} \mathrm{T}$ cell line to express a CAR. This CAR consisted of an anti-ErbB2 extracellular single chain variable fragment region linked to the intracellular signaling domain comprising CD28-CD3ל. The ability of three different promoters (CMV, Vav and LTR) to drive CAR expression was compared within each of the cell lines. CAR expression was highest under the CMV promoter in all cell lines, with expression ranging from $20-80 \%$. We subsequently performed functional analysis of these CMV-CAR expressing cells, and observed antigen-specific release of IFN- $\gamma$ from the $\mathrm{CD}^{+}$and $\mathrm{CD}^{+}{ }^{+} \mathrm{T}$ cell lines. In addition, antigen-specific release of both IL- 2 and IL-17 $\alpha$ was also detected from the CD4 ${ }^{+} \mathrm{T}$ cell line, EL4. Overall this investigation demonstrated the feasibility of electroporation to compare promoter activity, induce rapid expression and subsequent function of a CAR in a number of hematopoietic cell lines.
\end{abstract}

Keywords: Electroporation, genetic engineering, chimeric receptor, T cell, hematopoietic cells, vector screen, IL-17 $\alpha$.

\section{INTRODUCTION}

Adoptive immunotherapy is a promising treatment for cancer, which has been shown to be particularly effective against metastatic melanoma $[1,2]$. To date, $\mathrm{CD}^{+}$CTLs have been the main effector cell used in adoptive immunotherapy, however growing evidence supports the use of CTLs simultaneously co-transferred with $\mathrm{CD}^{+}$helper $\mathrm{T}$ cells (Th) [3-7]. The co-transfer of $\mathrm{CD}^{+} \mathrm{T}$ cells has been shown to amplify and sustain the actions of CTL effector cells, as well as enhance the infiltration and persistence of $\mathrm{CD}^{+} \mathrm{T}$ cell numbers in the tumor mass [8]. In addition, secretion of IL-2 by $\mathrm{CD}^{+}$Th cells has been found to stimulate the differentiation of naive $\mathrm{CD}^{+}$cells into both effector CTLs and long term $\mathrm{CD}^{+}$memory $\mathrm{T}$ cells [6], and shown to be integral in the survival of CTLs after a second encounter with the target antigen [9-12]. Furthermore, the co-transfer of specific $\mathrm{CD} 4^{+} \mathrm{T}$ cell subsets, demonstrated a greater antitumor effect than the co-transfer of general $\mathrm{CD}^{+} \mathrm{T}$ cells, inducing a significantly large population of tumor specific

*Address correspondence to this author at the Cancer Immunology Research Program, Sir Peter MacCallum Department of Oncology, University of Melbourne, Parkville, Victoria 3010 Australia; Tel: +613 96561177; Fax: +613 96561411; E-mail: michael.kershaw@petermac.org

$\wedge$ Contributed equally to this work
$\mathrm{CD}^{+} \mathrm{T}$ cells $(5-10 \%)$ which resulted in greater tumor reduction [13].

While the combination of $\mathrm{CD}^{+}$and $\mathrm{CD}^{+}{ }^{+} \mathrm{T}$ lymphocytes has shown great potential in adoptive immunotherapy, the anti-tumor functions of other immune cells expressing a chimeric antigen receptor has yet to be explored fully. It is apparent that re-directing tumor specificity through genetic modification with CARs can lead to an improved anti-tumor response. However, due to the limitations with current methods of genetic modification, it is difficult to transduce other innate and adaptive immune cells with a CAR.

Therefore, in order to determine the anti-tumor function of murine $\mathrm{T}$ cell lines in addition to a macrophage cell line, we utilized the rapid method of electroporation. Using electroporation, we aimed to compare the expression and function of a CAR when driven by three different promoters, CMV, Vav and LTR. The CMV and LTR promoters are viral promoters while the Vav promoter is endogenously expressed. In physiological conditions, the Vav promoter drives the expression of the Vav gene, a member of the family of guanine nucleotide exchange factors. The protein isoform Vav1 is expressed in a pan-hematopoietic manner and is crucial in the development of $\mathrm{B}$ and $\mathrm{T}$ lymphocytes and for function of Natural Killer cells [14-18]. 
While the CMV and LTR promoter are well characterized and commonly used in genetic modification [12, 19], few studies have demonstrated the ability of the Vav promoter to be used in genetic modification of immune cells. Previous studies have highlighted the ability of the Vav promoter to drive the expression of a transgene in an in vivo model [20, 21], however little is known about its ability to drive CAR expression in an in vitro context. Therefore, we generated a vector containing a CAR under the control of the Vav promoter in order to test its ability in an in vitro setting.

The CAR utilized in this study was comprised of a single chain variable fragment $(\mathrm{scFv})$ specific for erythroblastic leukemia viral oncogene homolog 2, ErbB2, also known as the tyrosine kinase-type cell surface receptor, Her-2. This was linked to an extracellular c-myc region (for detection of CAR expression), which was further joined to the intracellular signaling $\mathrm{CD} 28-\mathrm{CD} 3 \zeta$ tail. Basal expression of ErbB2 can be detected on epithelial cells in areas such as the lungs, breast, and ovaries, however upregulation of ErbB2 expression has been correlated with poor prognosis in malignancies including breast and ovarian cancer [22, 23].

In this study, we utilized electroporation to genetically modify a range of murine $\mathrm{T}$ cell lines in addition to a murine macrophage cell line to express a CAR. A comparison was made between the ability of three different promoters (CMV, LTR and Vav) to drive CAR expression. A selection of murine hematopoietic cell lines, in particular a macrophage, $\mathrm{CD}^{+} \mathrm{T}$ cell (Th1), CD4 ${ }^{+} \mathrm{T}$ cell (Th17) and $\mathrm{CD}^{+} \mathrm{T}$ cell line were transiently modified to express the CAR. Having established the expression of the receptor on each cell line, the degree of response from these genetically modified cells when cultured against antigen positive target cells was assessed using cytokine secretion assays.

\section{MATERIALS AND METHODS}

\section{Cell Lines}

All cell lines used in this study were obtained from the American Type Culture Collection (ATCC) (Manassas, VA, USA) with the exception of the murine sarcoma line, 24JK, (kindly provided by Dr. P. Hwu, National Institute of Health (NIH), Betheseda, MD) [24] and 14.1 (kindly provided by Dr. J.A. Berzofsky, NIH, Betheseda, MD)[25].

The murine macrophage cell line RAW 264.7 and murine T cell lymphoma EL4 were cultured in Dulbecco's modified eagles medium (DMEM) (Life Technologies, Gibco, Grand Island, NY) supplemented with 10\% heat-inactivated fetal calf serum (FCS) (MultiSer, Thermo Trace, Melbourne), 2 mM glutamine (Gibco), $0.1 \mathrm{mM}$ non-essential amino acids (NEAA) (Gibco), $1 \mathrm{mM}$ sodium pyruvate (Gibco), 100 $\mu \mathrm{g} / \mathrm{mL}$ streptomycin (Sigma-Aldrich, St Louis, MO) and 100 $\mathrm{U} / \mathrm{mL}$ penicillin (Sigma-Aldrich) in an incubator at $37^{\circ} \mathrm{C}$ with $10 \% \mathrm{CO}_{2}$.

The murine $\mathrm{CD}^{+} \mathrm{T}$ cell line 14.1 and murine sarcoma cell lines 24JK and 24JK-ErbB2 were cultured in Roswell Park Memorial Institute media (RPMI) (Gibco) with additives (as described above). The murine CD8 ${ }^{+}$CTL cell line, CTLL-2, was maintained in RPMI (Gibco) with additives and 20\% heat-inactivated FCS (MultiSer). Both CTLL-2 and 14.1 cells were supplemented with $50 \mathrm{IU} / \mathrm{mL}$ of human re- combinant interleukin-2 (rhIL-2) (Biological Resources Branch, National Cancer Institute, Frederick, MD) every second day.

\section{Plasmids}

The CAR expressing the scFv-anti-ErbB2-CD28-CD3 $\zeta$ receptor contained the extracellular scFv-anti-ErbB2 monoclonal antibody (mAb) region (kindly provided by Dr. Winfried Wels). The CMV-CAR (present in the PMAX plasmid) was generated by Dr. Hollie Pegram (Peter MacCallum Cancer Centre (PMCC), Victoria, Australia). The LTR-CAR (present in the pLXSN plasmid) was generated previously [26]. The CMV-GFP (present in the PMAX plasmid) was supplied by Lonza (Basel, Switzerland). The HS21/45 Vav hCD4 plasmid was generously provided by Dr. Jerry Adams (Walter and Eliza Hall Institute, Melbourne, Victoria, Australia). The CAR incorporated into the HS21/45 plasmid (containing the Vav promoter) was generated in our laboratory using standard molecular biology methods.

\section{Electroporation}

Transfection of the CAR plasmid into the selected cell lines was performed using the Nucleofector (Lonza). Specific optimized protocols for each cell line were used as per the manufacturer's instructions. Each specific program utilized the optimal voltages as determined by the manufacturer. Preliminary data regarding the expression of GFP in all cell lines utilized the Nucleofector Kit V (Lonza).

Briefly, $5 \times 10^{6}$ of each cell line was centrifuged at $90 \mathrm{~g}$ for 5 minutes, washed in PBS containing 0.5\% FCS and centrifuged at $90 \mathrm{~g}$ for 10 minutes. Cells were then resuspended in $100 \mu \mathrm{l}$ of Nucleofector solution (Kit V) and mixed with 10 $\mu \mathrm{g}$ DNA prior to transfer to a cuvette. EL4 cells were electroporated with the pre-optimized program C-009 and immediately plated into pre-warmed media with supplements in a 6-well plate. The same method was applied to RAW 264.7 cells using program D-032. Optimized protocols for CTLL-2 and 14.1 cell lines were not provided by the manufacturer, thus the program X-001 and C-009 were used respectively, as they were optimized for other murine $\mathrm{CD} 8^{+}$and $\mathrm{CD}^{+}$cell lines.

Low viability and poor DNA expression in both CTLL-2 and 14.1 cell lines prompted the comparison between programs within the Nucleofector $\mathrm{V}$ program and the $\mathrm{Nu}-$ cleofector Mouse T Cell Kit (Lonza), which is specifically optimized for both primary murine $\mathrm{T}$ cells and $\mathrm{T}$ cell lines. Similar to the Nucleofector Kit V protocol, 5 × $10^{6}$ cells of each cell line were resuspended in either Nucleofector Kit V solution or Nucleofector Mouse T Cell Kit solution. Four micrograms of CMV-GFP were added to each solution. Cells resuspended in Nucleofector Kit V solution were subjected to electroporation under the programs C-009 or X-001, both optimized by the manufacturer for two types of $\mathrm{CD}^{+} \mathrm{T}$ cell lines, EL4 (murine) and Jurkat (human) respectively. The cells were then added to $2 \mathrm{~mL}$ of pre-warmed RPMI with supplements. Cells resuspended in the Nucleofector Mouse T Cell Kit were electroporated using the program X-001 and added to $2 \mathrm{~mL}$ of pre-warmed Mouse T Cell Media with the 
addition of 5\% FCS, $1 \% 200 \mathrm{mM}$ glutamine, in addition to supplements provided by the manufacturer. Fifty international units (IU)/mL of IL-2 were added to each well every alternate day.

\section{Flow Cytometry}

Expression of the CAR and GFP was assessed using flow cytometry. Twenty microlitres of Fc block (2.4G2 hybridoma supernatant) was added to RAW264.7 cells 10 mins prior to antibody staining. Cells electroporated with the CAR were primarily stained with purified anti-c-myc antibody (Cell Signaling, Beverly MA, USA) or purified isotype antiIgG2a (BD Bioscience, San Jose, California USA). Cells were then stained with anti-mouse Ig conjugated to phycoerythrin (PE) (Chemicon, Melbourne, Australia).

Cells electroporated with GFP were washed and analyzed unstained. All flow cytometry was performed using the BD FACS Canto2 cytometer (Becton Dickinson and Company) and FlowJo software (Tree Star Inc, Ashland OR). Unless otherwise indicated, flow cytometry for experiments over a 5 day period were taken at the following timepoints post transfection; Day 1: 6-9 hours, Day 2: 24 - 31 hours, Day 3: 48 52 hours, Day 4: 72 - 76 hours, Day 5: 96 - 101 hours.

\section{ELISA and Cytometric Bead Array (CBA)}

Transfected cells expressing either CMV-CAR or CMVGFP were co-cultured with the following conditions; RPMI alone, phorbol myristate acetate (PMA) (Sigma) plus ionomycin (Sigma) as a positive control, purified anti-IgG2a or purified anti-c-myc antibody in addition to antigen negative and positive target tumor cells, 24JK and 24JK-ErbB2. Target cells were resuspended to a total of $1 \times 10^{6} / \mathrm{mL}$ in RPMI containing supplements and aliquoted into a 48 well plate at a total concentration of $2.5 \times 10^{5}$. Plates were incubated at $37^{\circ} \mathrm{C}$ with $5 \% \mathrm{CO}_{2}$ for one hour prior to the addition of effector cells. Transfected cells expressing the CAR or GFP were resuspended at the same concentration as target cells (1 x $10^{6} / \mathrm{mL}$ ) and aliquoted at a $1: 1$ ratio of target:effector cells.. The plates were incubated overnight at $37^{\circ} \mathrm{C}$ in $5 \%$ $\mathrm{CO}_{2}$. Following overnight incubation, the supernatant was harvested, and analyzed by ELISA using standards for either IFN- $\gamma$ (Pharmingen) or IL-2 (eBioscience). The absorbance was analyzed using a Benchmark microplate reader (BioRad, Hercules, CA, USA) at $405 \mathrm{~nm}$ with Softmax Pro software (Molecular Devices, Sunnydale, CA). Interleukin- $17 \alpha$ secretion from EL4 cells was determined by cytometric bead array (CBA) (BD Bioscience). Supernatants from coculture experiments using CMV-CAR and CMV-GFP transfected EL4 cells were used as samples in this experiment. Analysis was performed via flow cytometry.

\section{Statistical Analysis}

Unless otherwise stated, statistical significance for all experiments was determined using Student's unpaired t-test and Graphpad Prism (Graphpad Software, San Diego, California). The standard error of the mean (SEM) was calculated to produce error bars in graphs.

\section{RESULTS}

\section{Optimization of Expression of Transgenes in CTLL-2} and 14.1 Cell Lines by Electroporation

We first set out to determine the efficiency of electroporation for genetically modifying our range of immune cells. Previously, genetic modification with the anti-ErbB2-CD28CD3z receptor in our laboratory had utilized retroviral transduction, a method allowing for stable expression of the transduced gene. However, as DNA constructs larger than 5 $\mathrm{kb}$ are difficult to express by retroviral transduction[27], and given that production of retroviral vectors is time consuming, we used electroporation as an alternative method of genetic modification. Before assessing the expression and function of the CAR plasmid in various immune cells, we wanted to determine the ability of our selected immune cell lines to express DNA using the method of electroporation.

Using the reporter gene GFP, we electroporated the cell lines with their corresponding pre-set programs as determined by the manufacturer. Pre-set programs for the cell lines, EL4 and RAW 264.7 were provided, however programs for both CTLL-2 and 14.1 cell lines were not available. Therefore, we optimized the protocols for CTLL-2 and 14.1 using programs from the Nucleofector Kit V (Kit V), which is used for a general range of cells, and the Nucleofector Mouse T Cell Kit (Mouse T Cell Kit), specifically optimized for primary $\mathrm{T}$ cells as well as $\mathrm{T}$ cell lines.

We sought to compare the optimal $\mathrm{T}$ cell programs from both Kit $\mathrm{V}$ protocols optimized for immortal $\mathrm{T}$ cell lines to and the Mouse T Cell Kit for CTLL-2 (CD8 ${ }^{+} \mathrm{T}$ cell) and $14.1\left(\mathrm{CD}^{+} \mathrm{T}\right.$ cell) cell lines. The Nucleofector Kit V provided the pre-optimized program C-009 for electroporation of EL4 (a mouse CD4 ${ }^{+} \mathrm{T}$ cell line) and the pre-optimized program X-001 for Jurkat (a human $\mathrm{CD}^{+} \mathrm{T}$ cell line). The Mouse T Cell Kit utilized the program X-001. The cell lines were then electroporated with CMV-GFP plasmid DNA using all three programs to determine the optimal kit and program for the CTLL-2 and 14.1 cell line. Flow cytometry was performed on cells to determine the relative viability and GFP expression using all three programs (Figs. 1a and 2a). There was no significant difference in viability between Nucleofector programs. (Figs. $\mathbf{1 b}$ and $\mathbf{2 b}$ ).

As the level of DNA expression induced by each program was an important parameter, we also compared the expression of GFP in these cell lines through analyzing the mean fluorescence intensity (MFI) of the GFP positive population. In order to reduce the day to day variation, the MFI of GFP positive cells was normalized to the corresponding MFI of GFP expressing cells generated using the C-009 program. Using this method of analysis, we demonstrated that both CTLL-2 and 14.1 cell lines displayed significantly increased GFP expression $(\mathrm{P}(14.1)<0.02$, $\mathrm{P}(\mathrm{CTLL}-2)<0.01)$ using the Nucleofector Mouse T Cell Kit (Fig. 1c and 2c).

We observed a similar trend when observing the proportion of GFP positive population (normalized to the GFP positive population observed with the program C-009) (Figs. 1d and 2d). This suggested that the Mouse T Cell Kit provided greater transduction efficiency than the Kit $\mathrm{V}$ for both CTLL-2 and 14.1 cell lines. Therefore, all subsequent ex- 
(a)

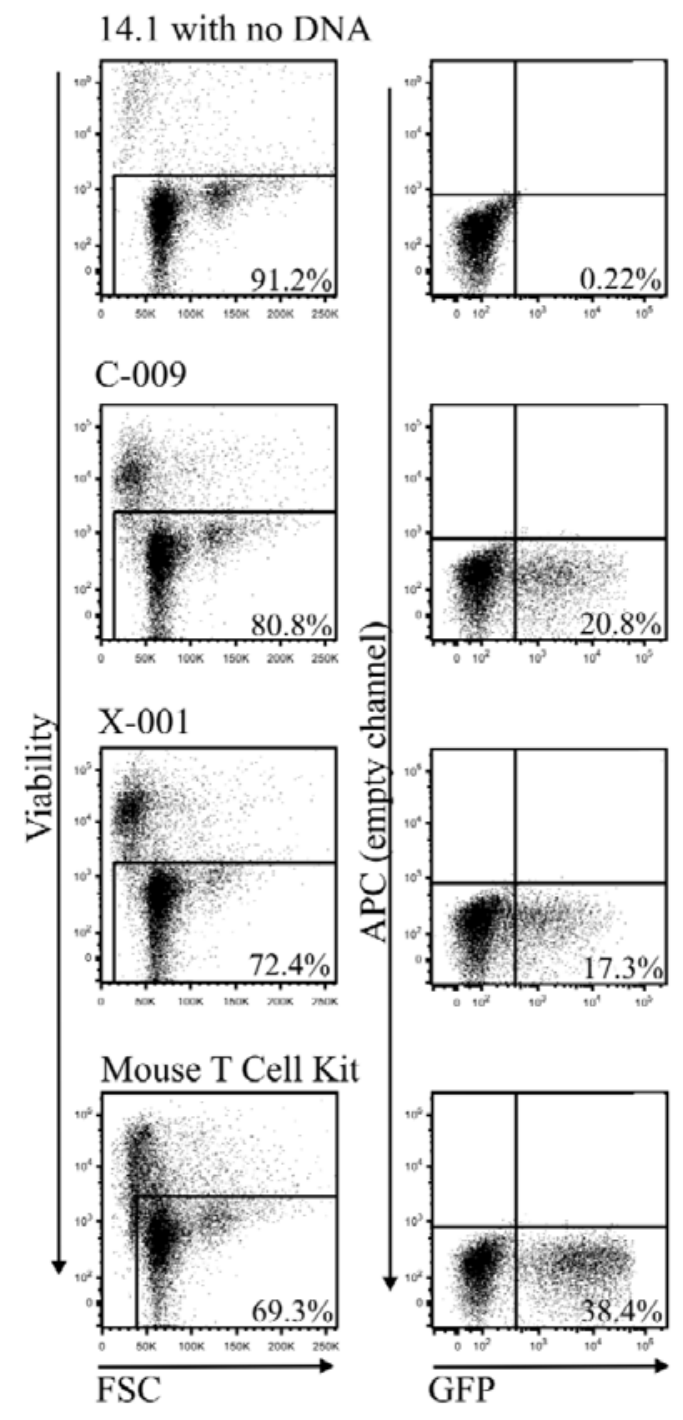

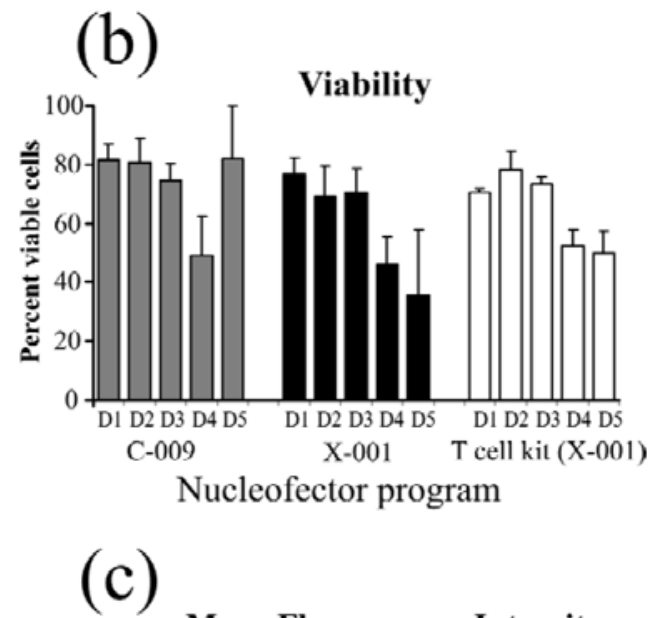

Mean Fluorescence Intensity

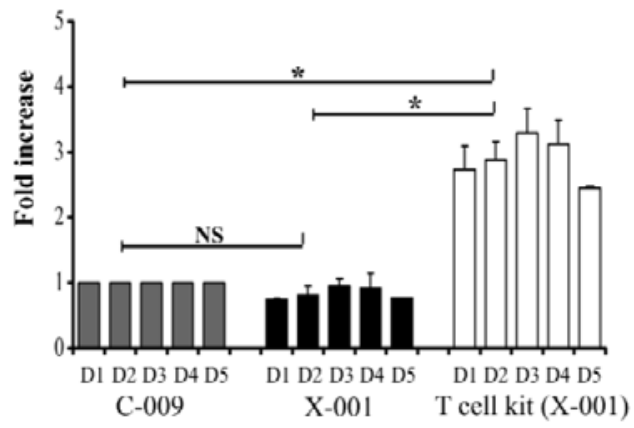

Nucleofector program

(d)

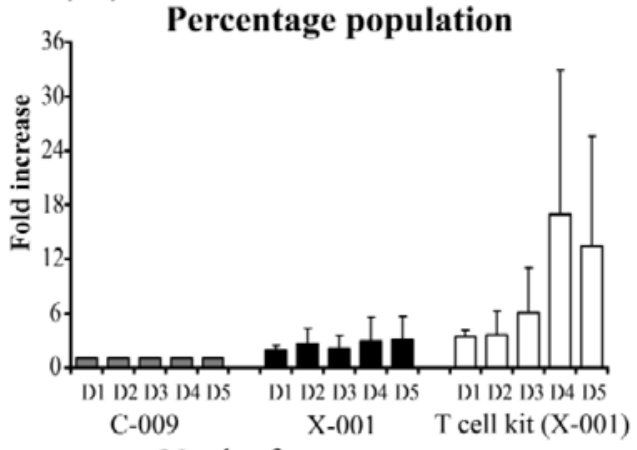

Nucleofector program

Fig. (1). Optimal expression of GFP in 14.1 cells was achieved using the Nucleofector Mouse T Cell Kit. 14.1 cells were electroporated with CMV-GFP with the indicated Nucleofector programs (refer to Methods and Materials). GFP expression was assessed via flow cytometry over a period of 5 days (D1-D5). Data shown is a combination of three independent experiments. (a) Representative graphs showing viability and GFP expression of cells generated with each specified program on day 2. (b) Percentage of viable cells for each Nucleofector program. (c) Mean fluorescence intensity of GFP positive populations were analyzed for each program and normalized to the lowest expressing condition (C-009) on each day. Statistical significance of GFP expression between different programs was analyzed at day 2. (*P $<0.02$, NS = Not significant, Student's unpaired t-test). (d) Percentage of GFP ${ }^{+}$cells gated on total viable cells and normalized to the lowest expressing condition (C-009). Error bars represent SEM.

periments involving CTLL-2 and 14.1 cell lines were performed using the Mouse T Cell Kit.

\section{DNA Expression in Electroporated Cell Lines is Optimal at 24 Hours}

Having determined the optimized protocol for each individual cell line, the next aim in our study was to determine the timepoint of optimal expression in each cell line. Cell lines were electroporated with CMV-GFP and the level of GFP expression was tracked over a 5 day period (Fig. 3). While the level of GFP expression was relatively similar between all cell lines from day 2 onwards, there was a significant increase in GFP expression between day 1 and day 2 for EL4 and 14.1 cell lines (P(EL4) <0.01, P(14.1) <0.04, Student's unpaired t-test). In addition, the viability of the 
(a)

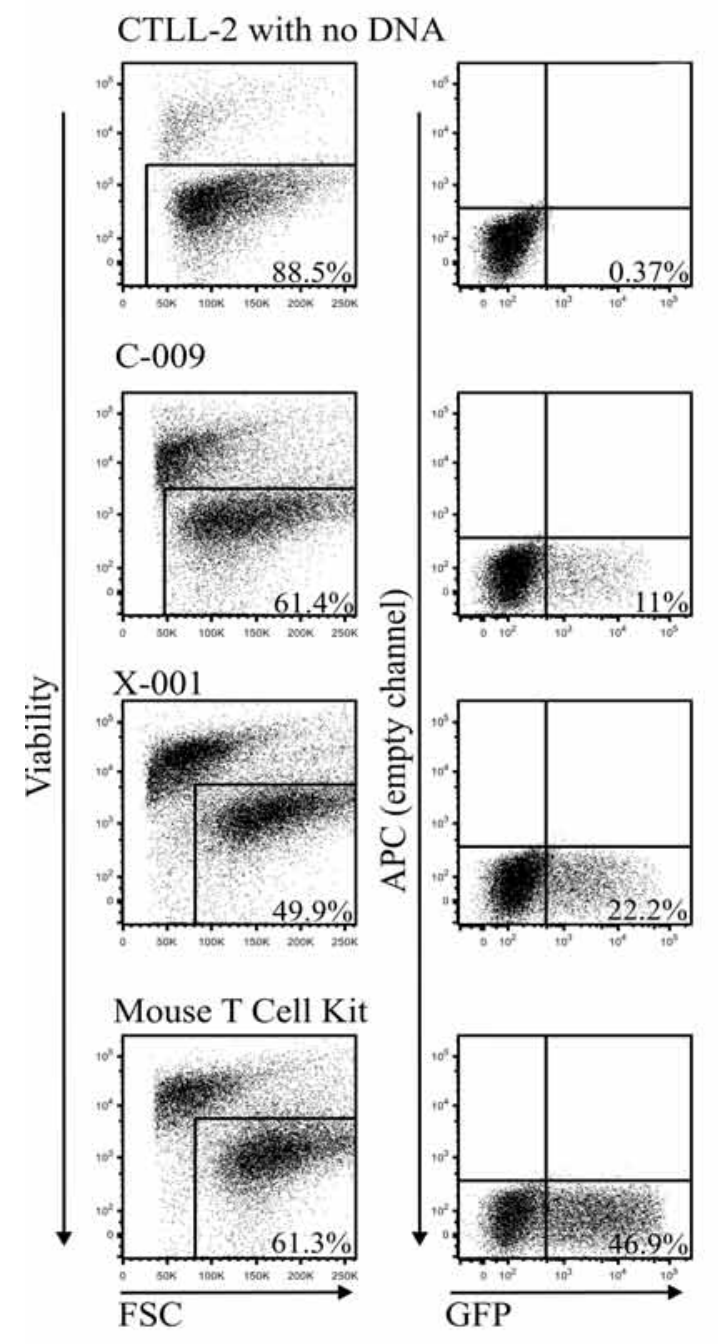

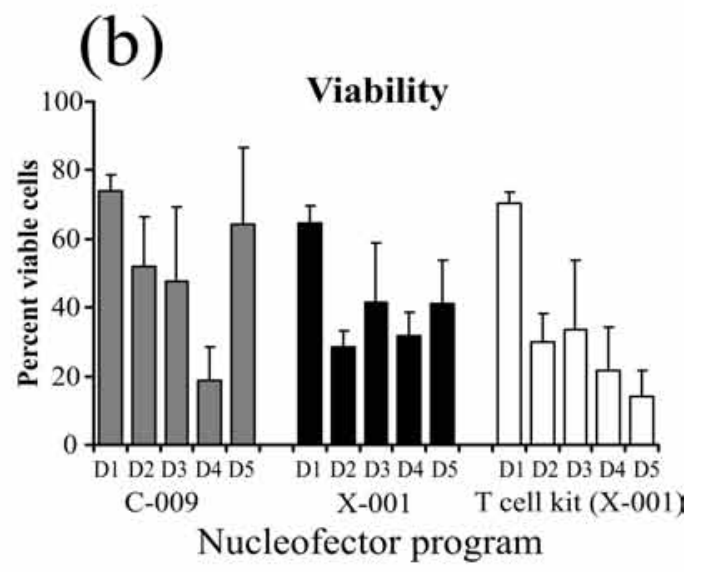

(c) Mean Fluorescence Intensity

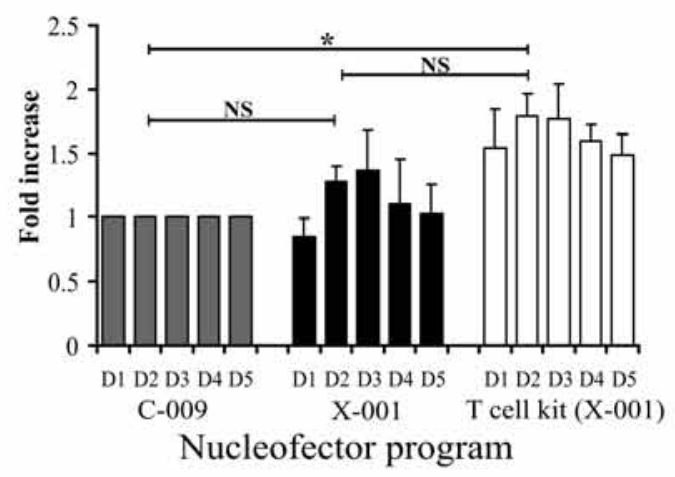

(d)

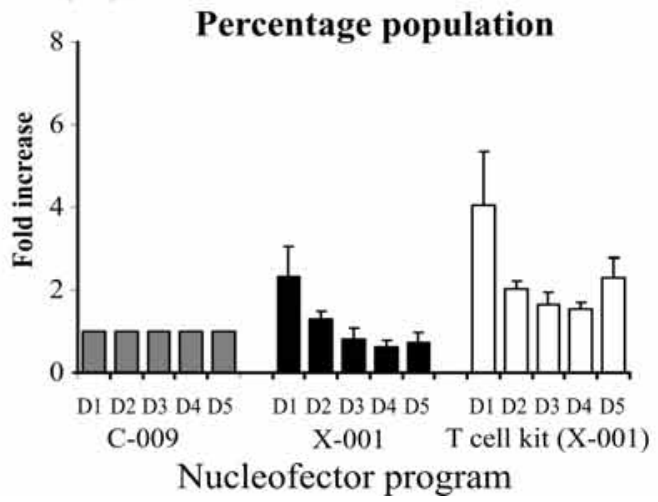

Fig. (2). Optimal expression of GFP in CTLL-2 cells was achieved using the Nucleofector Mouse T Cell Kit. CTLL-2 cells were electroporated with CMV-GFP with the indicated Nucleofector programs (refer to Methods and Materials). GFP expression was assessed via flow cytometry over a period of 5 days (D1-D5). Data shown is a combination of three independent experiments. (a) Representative graphs showing viability and GFP expression of cells generated with each specified program on day 2. (b) Percentage of viable cells for each Nucleofector program. Statistical significance of GFP expression between different programs was analyzed at day 2 . (*P $<0.01$, NS $=$ Not significant, Student's unpaired t-test). (c) Mean fluorescence intensity of GFP positive populations was analyzed for each program and normalized to the lowest expressing condition (C-009). (d) Percentage of $\mathrm{GFP}^{+}$cells gated on total viable cells and normalized to the lowest expressing condition (C-009). Error bars represent SEM.

GFP-expressing cells was observed to be greater at day 2 compared to day 1 (data not shown). For that reason, we decided that day 2 was the optimal timepoint for analysis of expression and subsequent functional analysis.

\section{Expression of CARs is Highest when Driven by the CMV Promoter}

After identifying the optimal electroporation protocols and timepoint for transgene expression using GFP driven by 
the CMV promoter, we next determined the ability of the different promoters to drive CAR expression. We initially chose to utilize one reporter cell line EL4 as it had the highest level of GFP expression over the 5 day period (Fig. 3) and in addition was more viable post-electroporation than the other cell lines.
EL4 cells were electroporated with vectors containing the CMV-CAR, LTR-CAR and Vav-CAR as well as CMV-GFP as a positive control. CAR expression was then analyzed using flow cytometry over a 5 day period. In the representative histogram (Fig. 4), the expression of GFP remained high throughout the 5 day period, ranging from $49.05 \pm 12.01$ to

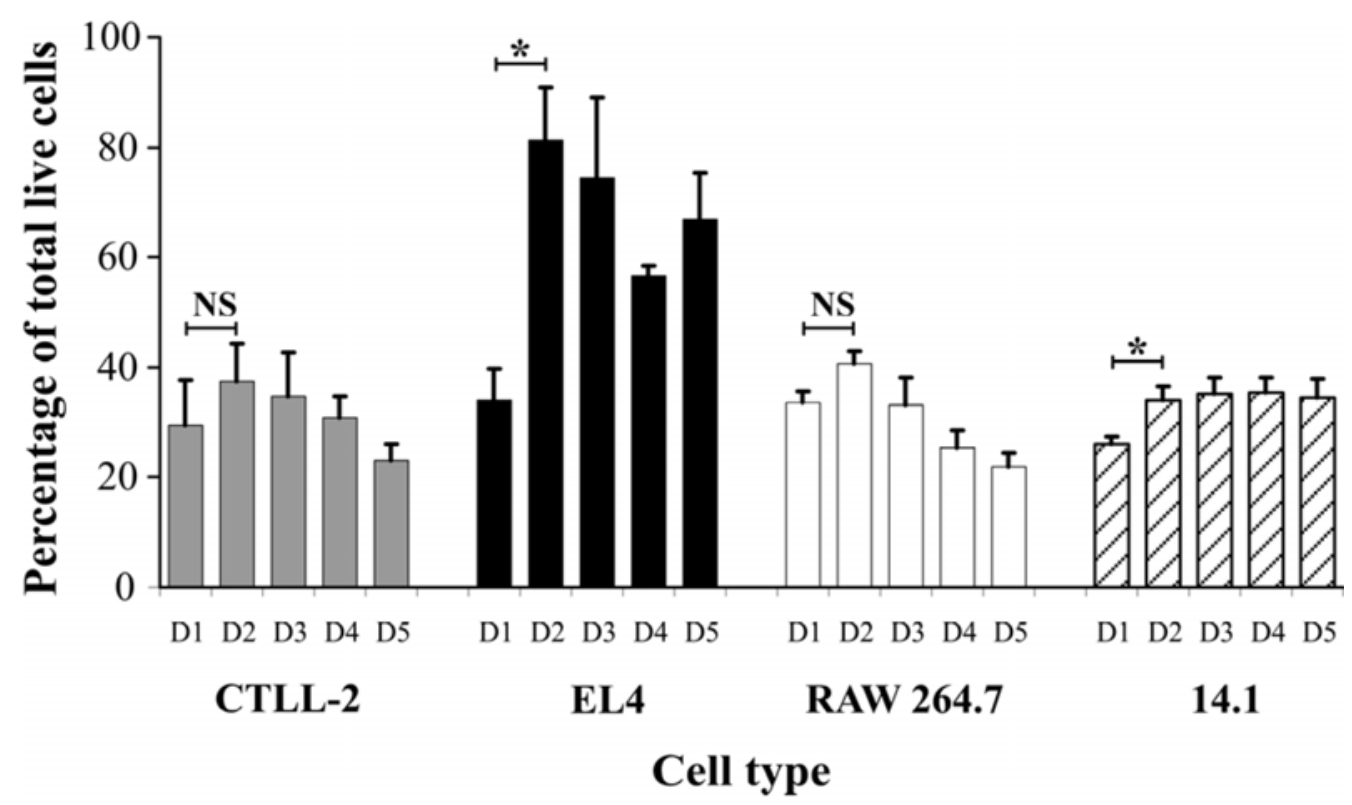

Fig. (3). Kinetic analysis of GFP expression in transfected cells over a 5 day timepoint. The indicated cell lines were electroporated with CMV-GFP using the Nucleofector instrument (refer to Methods and Materials) and incubated over a 5 day period. (a) GFP expression was assessed via flow cytometry and represented as a percentage of total live cells as indicated (D1-D5). Expression in EL4 and 14.1 cell lines is significantly greater at day 2 compared to day 1 . Data shown is a combination of three independent experiments. Error bars represent SEM. $(\mathrm{P}(\mathrm{CTLL}-2)<0.5, \mathrm{P}(\mathrm{EL} 4)<0.01, \mathrm{P}(\mathrm{RAW} 264.7)<0.09, \mathrm{P}(14.1)<0.04$, NS = Not significant, Student’s unpaired t-test).

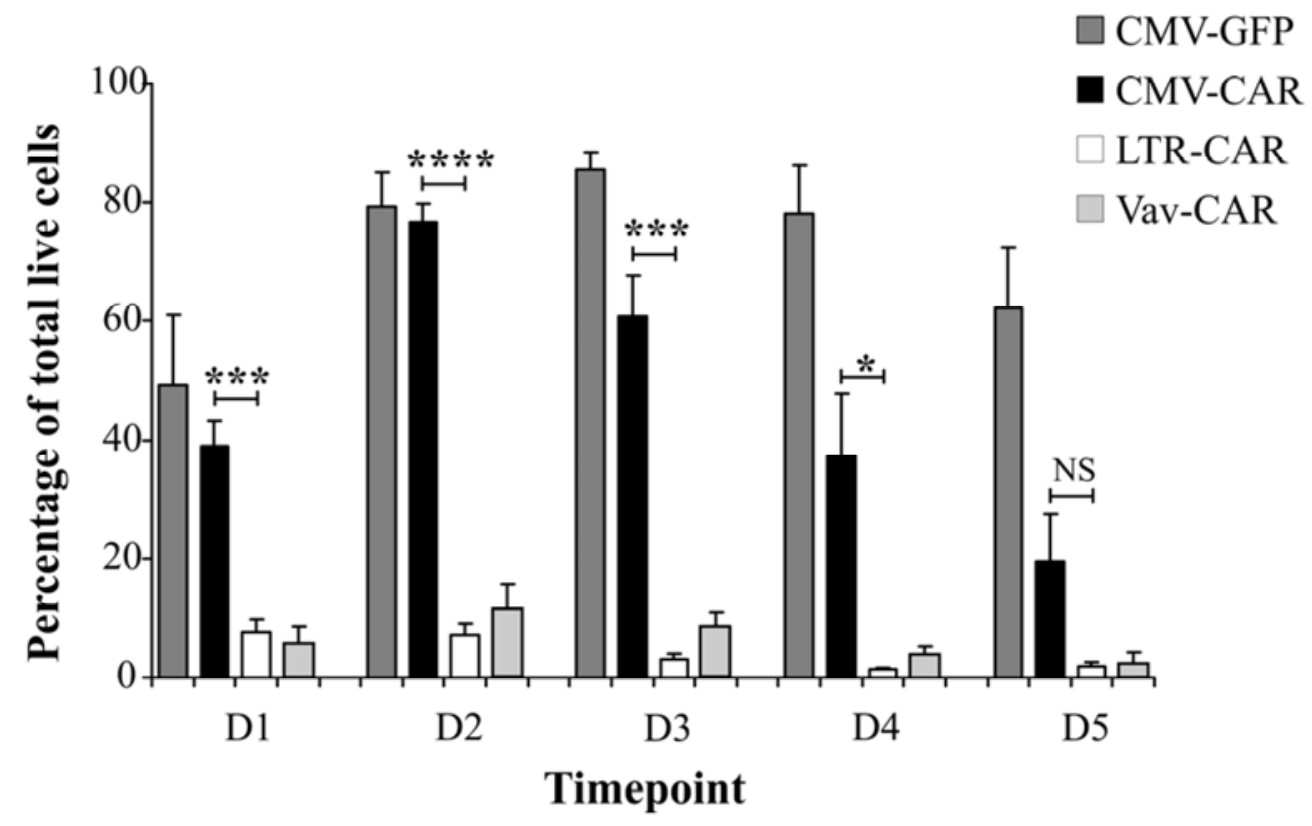

Fig. (4). Chimeric antigen receptor expression is highest under the CMV promoter in EL4 cells. EL4 cells were electroporated with DNA as indicated in the figure key, using Nucleofector program C-009. CMV GFP was used as a positive control for the CMV promoter. DNA expression was assessed via flow cytometry over a period of 5 days and represented as a percentage of total live cells as indicated (D1D5). DNA driven by the CMV promoter is shown to induce high levels of expression when compared to both LTR and Vav driven DNA. Data shown is a combination of four independent experiments. Error bars represent SEM. (P(D1) $<0.0006, P(D 2)<0.0001, P(D 3)<0.0002$, $\mathrm{P}(\mathrm{D} 4)<0.01, \mathrm{P}(\mathrm{D} 5)<0.09$, NS = Not significant, Student's unpaired t-test). 
$85.55 \pm 2.85$ (mean percentage of the positive population (Mean) \pm standard error of mean (SEM)). The expression of the CAR under the same promoter (CMV) was observed to be greater than both the LTR and Vav promoters, with maximum expression at the day 2 timepoint (76.65 \pm 3.05 , Mean \pm SEM). The degree of CAR expression under the LTR and Vav promoters was minimal in comparison to the CMV promoter at all time points (LTR-CAR $4.34 \pm 0.85$, Vav-CAR $6.56 \pm 1.35$, Mean of 5 timepoints \pm SEM).

This data suggests that the highly active CMV promoter was successful in driving the expression of the CAR in EL4 cells. As the main aim of our study was to use electroporation as a rapid means to determine the function of a range of hematopoietic cell lines expressing the anti-ErbB2 CAR, the next step was to validate the ability of our cell lines to ex- press a functional CAR. Having established the high level of activity of the CMV promoter in EL4 cells, we utilized the CMV-CAR to induce expression of the CAR in the other cell lines. In addition, as the ability of the Vav promoter had shown high levels of expression in both previous in vivo analyses [20, 21], we investigated whether the low expression of the Vav-CAR was specific for the EL4 cell line (Fig. 4) and whether this low expression extended to the other cell lines. We then electroporated our range of cells with both CMV-CAR and Vav-CAR DNA and observed the level of CAR expression over a period of 5 days (Fig. 5).

We observed a consistently higher level of CAR expression under the CMV promoter in comparison to the Vav promoter throughout our range of cells (Fig. 5), shown to be significant at day 2 for three of the four cell lines (P(CTLL-

(a)
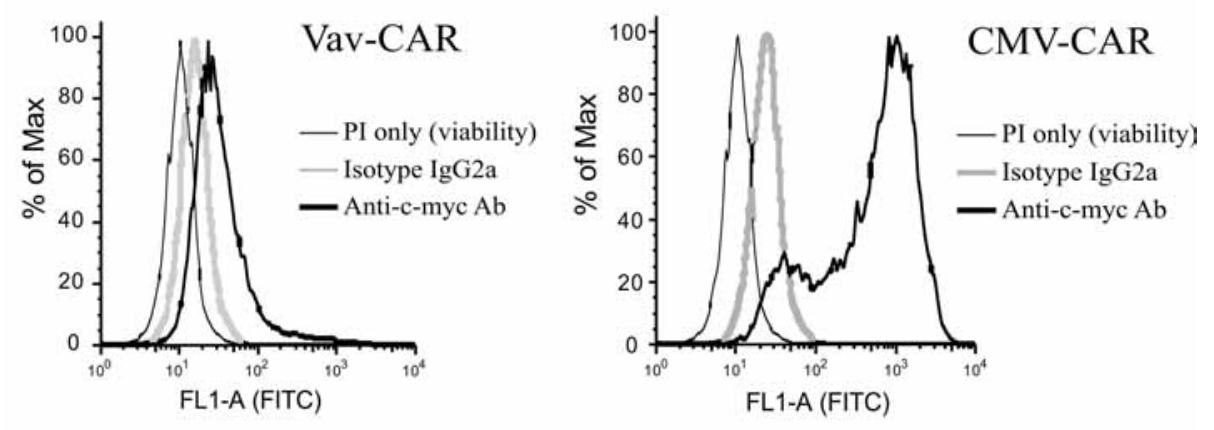

(b)

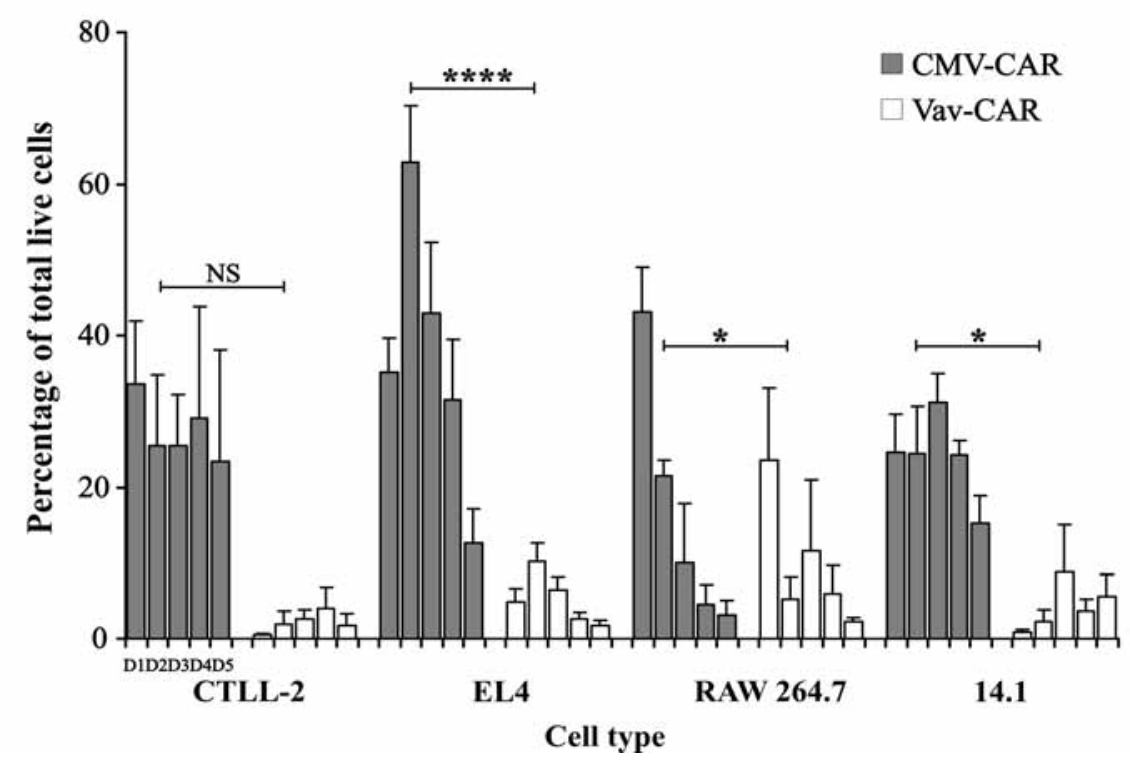

Fig. (5). The CMV promoter facilitates higher expression of a CAR in several hematopoietic cell lines over a 5 day period in comparison to the Vav promoter. Each indicated cell line was electroporated with CMV-CAR or Vav-CAR plasmid DNA. CAR expression was assessed via flow cytometry. (a) Representative plots of EL4 cells with no stain (thin line), isotype control (gray line), anti c-myc antibody (thick line). (b) The graph depicts CAR expression above that of isotype control over a period of 5 days and is represented as a percentage of total live cells as indicated (D1-D5). Statistical significance of DNA expression between CARs driven by the CMV promoter and Vav promoter was analyzed at day 2. Data shown is a combination of three independent experiments. Error bars represent SEM. (P(CTLL-2) $<0.07, \mathrm{P}(\mathrm{EL} 4)<0.0001, \mathrm{P}(\mathrm{RAW} 264.7)<0.01, \mathrm{P}(14.1)<0.02$, NS = Not significant, Student’s unpaired t-test). 
2) $<0.07$, P(EL4) <0.0001, P(RAW 264.7) <0.01, P(14.1) $<0.02$, Student's unpaired t-test). Although the expression of CMV-CAR was higher at day 2 for only EL4 cells, taking into account the viability of the cells, we decided to continue our functional studies at day 2. Consistent with the expression of the Vav-CAR in EL4 cells, we observed a similar low level of Vav-CAR in the other cell lines, indicating that although the Vav promoter was able to drive a low level of CAR expression in a range of cell lines, the level observed was significantly lower than the CMV promoter. As the MFI of the CMV-CAR was significantly greater than the VavCAR at all time points observed, we utilized the CMV-CAR to perform all subsequent functional analysis.

\section{CAR-expressing Cells Exhibit Antigen-specific Function Following Receptor Ligation}

After establishing the expression of CMV-CAR in our cell lines, we aimed to determine the response of these transfected effector cells against target cells expressing the ErbB2

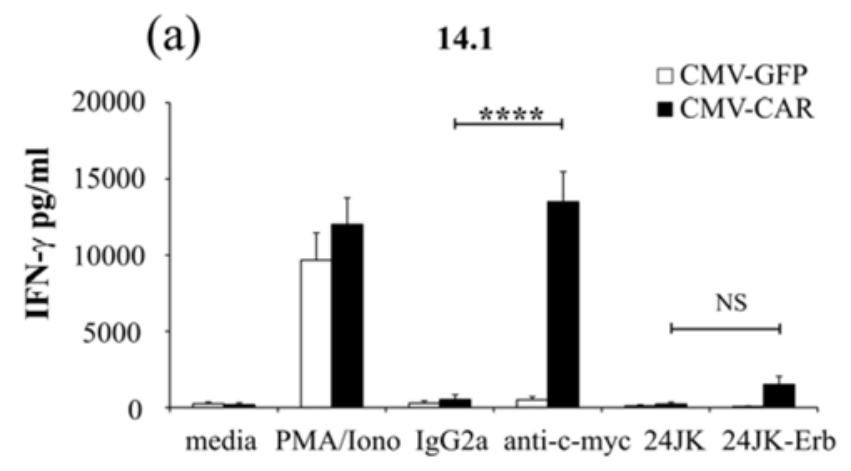

(b)

CTLL-2

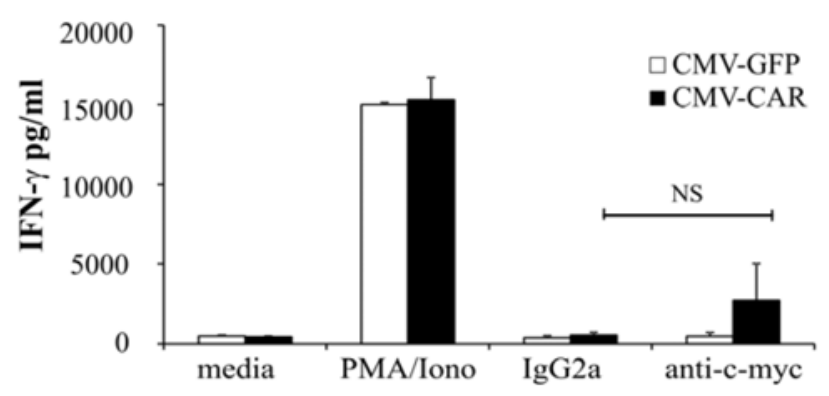

CTLL-2

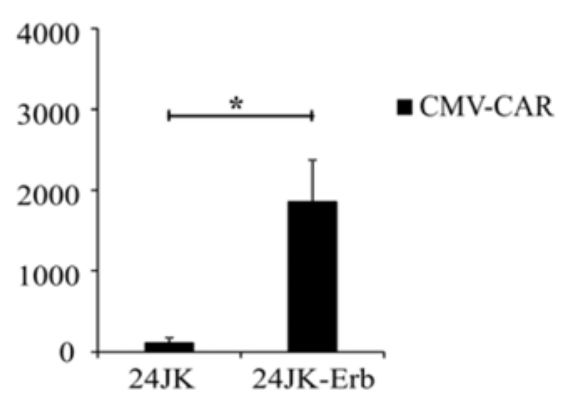

(c)

EL4

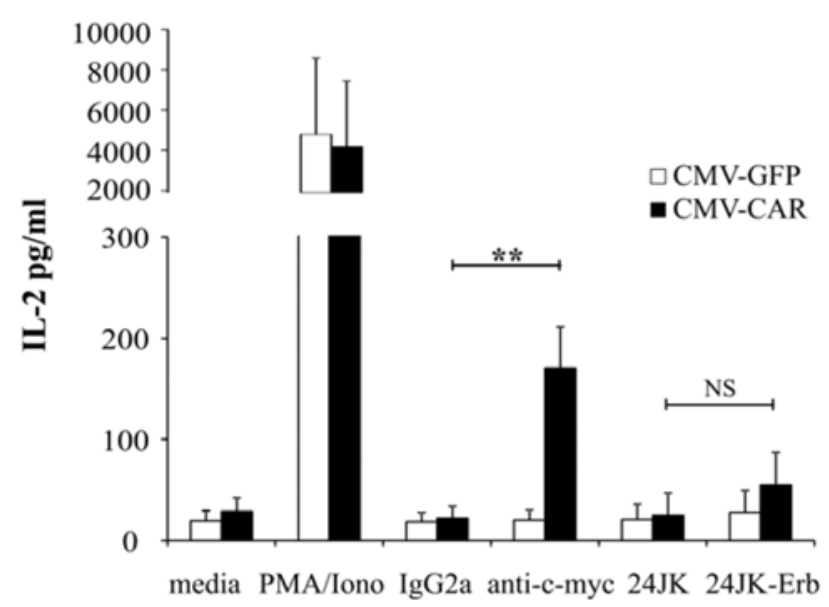

Fig. (6). CAR ligation induces antigen-specific cytokine secretion from immune cells. Specific cytokine secretion from (a) 14.1 cells, (b) CTLL-2 and (c) EL4 cells. These cells were electroporated with CMV-GFP or CMV-CAR and co-cultured with the indicated conditions. Target cells, 24JK and 24JK-ErbB2, were cultured in a 1:1 ratio with effector cells. After overnight co-culture, analysis of the harvested supernatant was performed using ELISA. Data shown is a combination of (a) 8 experiments (b) 2 - 5 experiments and (c) 6 experiments. Statistical analysis was used to compare the level of cytokine secretion between anti-c-myc antibody (tag) and IgG2a conditions, and 24JK and 24JK-ErbB2 conditions. (**** $\mathrm{P}<0.0001$, ** $\mathrm{P}<0.005$, * $\mathrm{P}<0.01$, NS $>0.05$ (not significant), Student's unpaired t-test). Error bars represent SEM. PMA/Iono = positive control stimulation by PMA plus ionomycin (see Methods). 
antigen. As IFN- $\gamma$ is an important cytokine in immune activation, and is secreted from $\mathrm{T}$ lymphocytes in response to antigen recognition $[19,28]$, we regarded the release of IFN$\gamma$ in response to receptor ligation as an indicator of $\mathrm{T}$ cell activation in our study.

14.1 cells expressing the CAR were able to significantly secrete IFN- $\gamma$ upon receptor ligation with the plate bound immobilized anti-c-myc antibody in comparison to both isotype IgG2a and GFP-expressing cells as assessed by ELISA (Fig. 6a) $(\mathrm{P}<0.0001$ (CMV-CAR anti-c-myc vs. CMV-CAR IgG2a). Significance did not extend to CAR-expressing cells co-cultured with 24JK-ErbB2. However, a significant increase in IFN- $\gamma$ secretion was observed when CTLL-2 cells, transduced with CMV-CAR, were co-cultured with 24JKErbB2 compared with 24JK (Fig. 6b).

Interleukin-2, another major cytokine in $\mathrm{T}$ lymphocyte biology has been shown to facilitate the growth and proliferation of $\mathrm{T}$ lymphocytes, in particular in response to antigen stimulation [29-31]. We observed antigen-specific IL-2 secretion by EL4 cells when co-cultured with the immobilized antibody anti-c-myc tag (Fig. 6c) $(\mathrm{P}<0.005$ (CMV-CAR antic-myc vs. CMV-CAR IgG2a), but not when co-cultured with target cells 24JK-ErbB2. We also investigated the secretion of IL-17 $\alpha$ from EL4 cells and observed antigen-specific release of IL-17 $\alpha$ when stimulated with both immobilized antibody anti-c-myc tag, as well as antigen positive target cells 24JK-ErbB2 (Fig. 7) (11.1 $\pm 1.5 \mathrm{ng} / \mathrm{ml}$ anti-c-myc vs. $2.0 \pm$ $0.3 \mathrm{ng} / \mathrm{ml} \mathrm{IgG2a} \mathrm{P}<0.0002$, and $3.7 \pm 0.6 \mathrm{ng} / \mathrm{ml}$ for $24 \mathrm{JK}-$ ErbB2 compared to $2.1 \pm 0.4 \mathrm{ng} / \mathrm{ml}$ for $24 \mathrm{JK} \mathrm{P}<0.04$, Mean \pm SEM). Importantly, we demonstrated through using the method of electroporation, the ability of cell lines transduced with the CMV-CAR to secrete cytokine in an antigenspecific manner, against both immobilized antibody and an- tigen positive target cells. (Fig. 6 and 7).

\section{DISCUSSION}

Immunotherapy is a promising treatment for cancer. Adoptive immunotherapy, in particular, has been demonstrated to be effective in treatment of cancers such as melanoma and viral associated malignancies [32, 33]. Furthermore, adoptive immunotherapies have also been tested in other types of cancer such as leukemia and neuroblastoma, in both mouse models and clinical trials [33-36]. While the use of genetically modified $\mathrm{CD}^{+}$and $\mathrm{CD}^{+} \mathrm{T}$ lymphocytes together has been shown to enhance their activity and cytotoxicity in mice [9, 37], the combination of other immune cells bearing a CAR has yet to be tested. In addition, the antitumor effector functions of many innate immune cells when expressing a CAR have yet to be fully characterized.

One of the most commonly used methods to genetically modify $\mathrm{T}$ cells is through gene insertion using retroviral transduction. Previous genetic modification with the CAR in our laboratory has been performed using retroviral transduction, which facilitated stable expression of the CAR in both murine and human T cells. However, this requires a fairly arduous process of retroviral production, determining adequate virus titer and optimizing transduction of the $\mathrm{T}$ cells followed by functional assessment of the CAR. In addition, the efficiency of retroviral transduction relies on the rapid multiplication of cells, which could lead to some difficulties with slower proliferative cells. Furthermore, it is difficult to express DNA vectors larger than 5kb [27] using this form of genetic modification. As the Vav-CAR plasmid used in our study was $11 \mathrm{~kb}$, we sought to optimize the alternative method of electroporation to genetically modify our cells with a large sized vector.

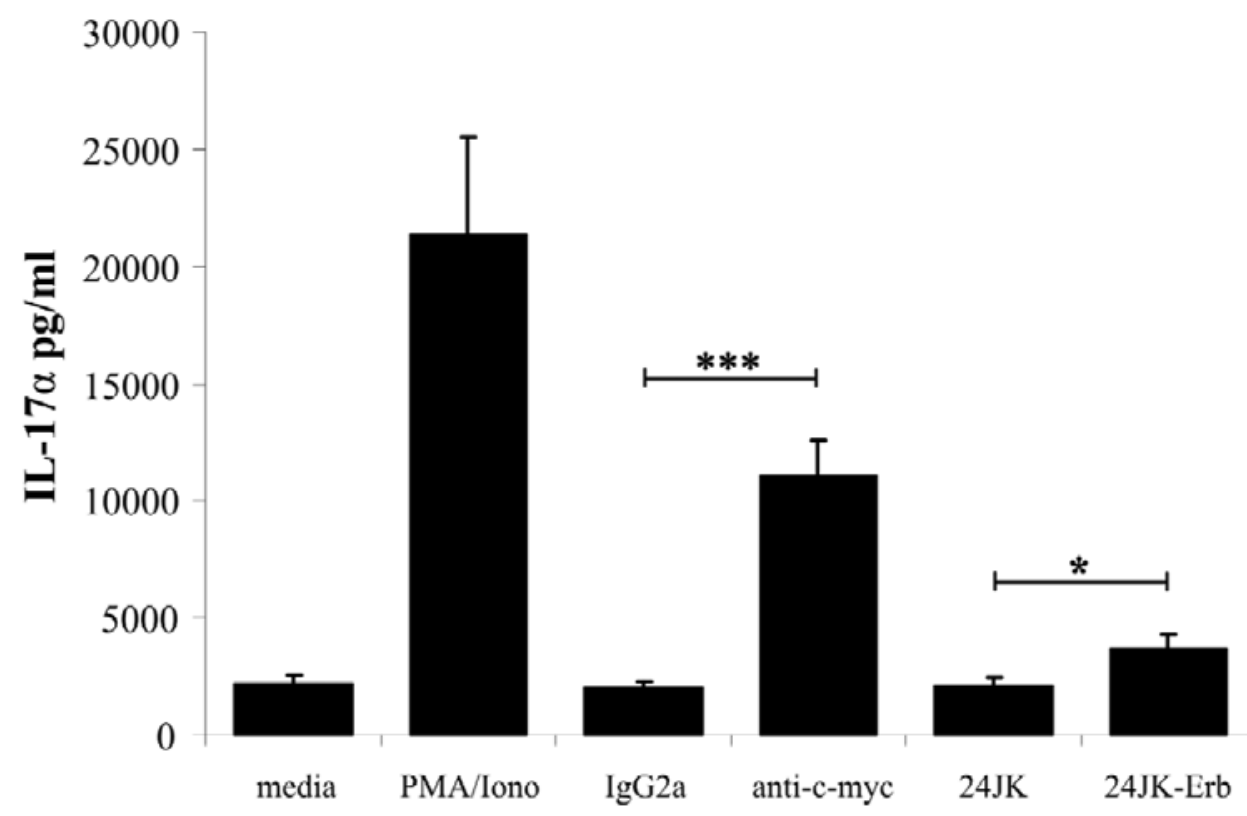

Fig. (7). CAR-expressing EL4 cells secrete IL-17 $\alpha$ in an antigen-specific manner. EL4 cells were electroporated with CMV-CAR and incubated overnight in the indicated combinations. Target cells, 24JK and 24JK-ErbB2, were cultured in a 1:1 ratio with effector cells. The supernatant was harvested and the level of cytokine present assessed using CBA. Data shown is a combination of 6 independent experiments. Error bars represent SEM. ( $* * * \mathrm{P}<0.0002$, * $\mathrm{P}<0.04$, Student's unpaired t-test). PMA/Iono = positive control stimulation by PMA plus ionomycin (see Methods). 
We also aimed to compare the ability of three different promoters to drive the expression of a CAR using the method of electroporation. Firstly, we optimized the protocols for each cell line and demonstrated the ability of our cell lines to express the transgene GFP (Fig. 3). We utilized flow cytometry to determine relative expression levels of GFP, since this was more sensitive than other methods such as immunofluorescence microscopy. Nevertheless, it is important to realize that other assays such as Western blotting may be useful to provide additional or confirmatory information of protein expression levels, particularly in studies of expression of non-fluorescent intracellular proteins.

We then sought to compare the level of CAR expression under the control of the Vav, LTR and CMV promoters (Figs. 4, 5a and 5b). Low expression of the CAR under the control of the Vav promoter was observed in all cell lines (Fig. 4 and 5b), despite having verified the expression of the Vav protein via Western Blot (data not shown). Although the ability of the Vav promoter to drive the expression of a transgene (hCD4 and Bcl-2) had been well-characterized in vivo [20, 21], its capabilities in an in vitro setting are unknown. Moreover, while the function of the Vav protein is important in hematopoiesis in a physiologically setting, in particular the development of both B and T lymphocytes [14, $16,17]$ our study utilized immortalized cell lines. Unlike naïve murine cells, immortalized cell lines can be presumed to have undergone multiple divisions and may present in what is expected to be a "terminally differentiated" state. The combination of these factors may account for the low expression observed in our results.

Previous results from our laboratory [12] have shown high level of CAR expression using the retroviral pLXSN plasmid containing the LTR-CAR in in vitro and in vivo experiments. Surprisingly, we observed a low level of CAR expression under the control of the LTR promoter (Fig. 5). However, as the function of the LTR viral promoter requires transgene integration into genome, the transient method of electroporation may not have facilitated for the proper activity of the LTR promoter.

While empty plasmids lacking the CAR gene were not included in figures 4 and 5, the kinetic comparison of CAR expression between time points remains valid. In addition, we have not previously observed non-specific binding of the CAR-specific antibody to cells transduced with the pLXSN plasmid lacking the CAR gene, and therefore we consider it unlikely that there was any significant contribution to antimyc staining from plasmid components other than the CAR gene.

The differences in the size of the plasmids themselves also may have had an effect on the level of expression. The PMAX plasmid (CMV promoter) showed the highest expression, with a total size of roughly $4.5 \mathrm{~kb}$. In contrast, both pLXSN plasmid (LTR promoter) and HS21/45 plasmid (Vav promoter) showed little expression, with sizes of $7.5 \mathrm{~kb}$ and $11 \mathrm{~kb}$ respectively. The low expression observed in both LTR and Vav driven plasmids may have resulted from the large size of the plasmids in addition to the ability and strength of each promoter. Furthermore, although equal amounts of each DNA plasmid were included in the electroporation procedure, it should be noted that the plasmid backbones varied.
This may have resulted in differing amounts of DNA entering cells, which may also have contributed to the differing expression levels of proteins.

It was necessary to restrict our analysis of CAR expression to cell surface protein using flow cytometry, since this is where CARs exert their function. However, studies into the expression of intracellular proteins would benefit from additional assays such as Western blot.

Having established the protocol for optimal expression of the CMV-CAR in various cell lines, we performed functional studies to determine the ability of the transduced cell lines to recognize antigen-expressing target cells. Antigen-specific release of IFN- $\gamma$ was observed from both 14.1 and CTLL-2 cells whereas IL- 2 and IL-17 $\alpha$ secretion was detected from EL4 cells when transduced with the CMV-CAR. The corresponding GFP transfected cells were found to secrete similar levels of cytokine in response to PMA/Ionomycin stimulation however no response was observed against both anti-cmyc antibody and 24JK-ErbB2, indicating the antigenspecific cytokine release was mediated through the CAR.

A marked secretion of both IFN- $\gamma$ from 14.1 cells as well as IL-2 and IL-17 $\alpha$ from EL4 cells was observed in response to the immobilized antibody anti-c-myc compared to the levels detected in response to the target cell 24JK-ErbB2. The concentration of immobilized antibody is generally much higher than the level of antigen expressed by the target cells, perhaps accounting for the large difference in cytokine secretion observed. In addition, the difference in the binding region of the c-myc antibody and the ErbB2 antigen on the CAR may have accounted for the difference in the level and intensity of receptor stimulation.

Overall, we determined that electroporation is a relatively quick and reliable method to transiently modify a number of $\mathrm{T}$ cell lines as well as a macrophage cell line. Using electroporation increases the efficiency and ease of selecting the best promoter able to drive transgene expression, allowing selection of the best promoter with which to perform future analysis utilizing more permanent genetic modification techniques. Our observations have relevance for future studies in adoptive immunotherapy. By screening a range of immune cells for their ability to express a functional CAR, we can select candidates suitable for stable genetic modification in a quick and rapid manner.

We have provided a screening method to select the best vector and promoter to transfect both innate and adaptive immune cells with a CAR, allowing for further functional analysis. Future studies about CAR bearing immune cells and their anti-tumoral potential may then be used to generate the most potent anti-tumoral combination of immune cells for adoptive immunotherapy.

\section{ACKNOWLEDGEMENTS}

This work was supported by a grant from the National Health and Medical Research Council of Australia (1006188)

\section{REFERENCES}

[1] Robbins PF, Morgan RA, Feldman SA, et al. Tumor regression in patients with metastatic synovial cell sarcoma and melanoma using 
genetically engineered lymphocytes reactive with ny-eso-1. J Clin Oncol 2011; 29: 917-24.

[2] Kerkar SP, Muranski P, Kaiser A, et al. Tumor-specific CD8+ T cells expressing interleukin-12 eradicate established cancers in lymphodepleted hosts. Cancer Res 2010; 70: 6725-34.

[3] Chamoto K, Kosaka A, Tsuji T, et al. Critical role of the Th1/Tc1 circuit for the generation of tumor-specific CTL during tumor eradication in vivo by Th1-cell therapy. Cancer Sci 2003; 94: 9248.

[4] Zhong XS, Matsushita M, Plotkin J, Riviere I, Sadelain M. Chimeric antigen receptors combining 4-1BB and CD28 signaling domains augment PI3kinase/AKT/Bcl-XL activation and CD8+ T cell-mediated tumor eradication. Mol Ther 2010; 18: 413-20.

[5] Carpenito C, Milone MC, Hassan R, et al. Control of large, established tumor xenografts with genetically retargeted human $\mathrm{T}$ cells containing CD28 and CD137 domains. Proc Natl Acad Sci USA 2009; 106: 3360-5.

[6] Marzo AL, Lake RA, Robinson BW, Scott B. T-cell receptor transgenic analysis of tumor-specific CD8 and CD4 responses in the eradication of solid tumors. Cancer Res 1999; 59: 1071-9.

[7] Cheadle EJ, Hawkins RE, Batha H, Rothwell DG, Ashton G, Gilham DE. Eradication of established B-cell lymphoma by CD19specific murine $\mathrm{T}$ cells is dependent on host lymphopenic environment and can be mediated by CD4+ and CD8+ T cells. J Immunother 2009; 32: 207-18.

[8] Dranoff G, Jaffee E, Lazenby A, et al. Vaccination with irradiated tumor cells engineered to secrete murine granulocyte-macrophage colony-stimulating factor stimulates potent, specific, and longlasting anti-tumor immunity. Proc Natl Acad Sci USA 1993; 90: 3539-43.

[9] Marzo A, Kinnear B, Lake R, et al. Tumor-specific CD4+ T cells have a major "post-licensing" role in CTL mediated anti-tumor immunity. J Immunol 2000; 165: 6047-55.

[10] Ossendorp F, Mengede E, Camps M, Filius R, Melief C. Specific T helper cell requirement for optimal induction of cytotoxic $\mathrm{T}$ lymphocytes against major histocompatability complex class II negative tumors. J Exp Med 1998; 187: 693-702.

[11] Goedegebuure P, Eberlein T. The role of CD4+ tumor-infiltrating lymphocytes in human solid tumours Immunologic Research 1995;14: 119-31.

[12] Moeller M, Kershaw M, Cameron R. Sustained antigen-specific antitumour recall response mediated by gene-modified CD4+ T helper-1 and CD8+T cells. . Cancer Res 2007; 67: 11428-37.

[13] Antony PA, Piccirillo CA, Akpinarli A, et al. CD8+ T cell immunity against a tumor/self-antigen is augmented by CD4+ T helper cells and hindered by naturally occurring $\mathrm{T}$ regulatory cells. J Immunol 2005; 174: 2591-601.

[14] Vigorito E, Gambardella L, Colucci F, McAdam S, Turner M. Vav proteins regulate peripheral B-cell survival. Blood 2005; 106: 2391-8.

[15] Katzav S, Martin-Zanca D, Barbacid M. vav, a novel human oncogene derived from a locus ubiquitously expressed in hematopoietic cells. EMBO J 1989; 8: 2283-90.

[16] Saveliev A, Vanes L, Ksionda O, et al. Function of the nucleotide exchange activity of vav1 in T cell development and activation. Sci Signal 2009; 2: ra83.

[17] Zhang R, Alt FW, Davidson L, Orkin SH, Swat W. Defective signalling through the $\mathrm{T}$ - and B-cell antigen receptors in lymphoid cells lacking the vav proto-oncogene. Nature 1995; 374: 470-3.

[18] Fujikawa K, Miletic AV, Alt FW, et al. vav1/2/3-null mice define an essential role for vav family proteins in lymphocyte development and activation but a differential requirement in mapk signaling in t and b cells. J Exp Med 2003; 198: 1595-608.
[19] Farrar MA, Schreiber RD. The molecular cell biology of interferon-gamma and its receptor. Annu Rev Immunol 1993; 11: 571-611.

[20] Ogilvy S, Metcalf D, Print CG, Bath ML, Harris AW, Adams JM. Constitutive Bcl-2 expression throughout the hematopoietic compartment affects multiple lineages and enhances progenitor cell survival. Proc Natl Acad Sci USA 1999; 96: 14943-8.

[21] Ogilvy S, Metcalf D, Gibson L, Bath ML, Harris AW, Adams JM. Promoter elements of vav drive transgene expression in vivo throughout the hematopoietic compartment. Blood 1999; 94: 185563.

[22] Slamon DJ, Godolphin W, Jones LA, et al. Studies of the HER$2 /$ neu proto-oncogene in human breast and ovarian cancer. Science 1989; 244: 707-12

[23] Press MF, Cordon-Cardo C, Slamon DJ. Expression of the HER2/neu proto-oncogene in normal human adult and fetal tissues. Oncogene 1990; 5: 953-62.

[24] Shiloni E, Karp SE, Custer MC, et al. Retroviral transduction of interferon-gamma cDNA into a nonimmunogenic murine fibrosarcoma: generation of $\mathrm{T}$ cells in draining lymph nodes capable of treating established parental metastatic tumor. Cancer Immunol Immunother 1993; 37: 286-92.

[25] Kurata A, Berzofsky JA. Analysis of peptide residues interacting with MHC molecule or T cell receptor. Can a peptide bind in more than one way to the same MHC molecule? J Immunol 1990; 144 4526-35.

[26] Haynes NM, Trapani JA, Teng MlWL, et al. Single-chain antigen recognition receptors that costimulate potent rejection of established experimental tumors. Blood 2002; 100: 3155-63.

[27] Miller AD. Development and Applications of Retroviral Vectors. In: Coffin JM, Hughes SH, Varmus HE, Eds. Retroviruses. Cold Spring Harbor NY: Cold Spring Harbor Laboratory Press 1997.

[28] Boehm U, Klamp T, Groot M, Howard JC. Cellular responses to interferon-gamma. Annu Rev Immunol 1997; 15: 749-95.

[29] Nelson BH. IL-2, regulatory T cells, and tolerance. J Immunol 2004; 172: 3983-8.

[30] Oppenheim JJ. IL-2: more than a T cell growth factor. J Immunol 2007;179:1413-4.

[31] Boyman O, Sprent J. The role of interleukin-2 during homeostasis and activation of the immune system. Nat Rev Immunol 2012; 12: 180-90.

[32] Dudley ME, Yang JC, Sherry R, et al. Adoptive cell therapy for patients with metastatic melanoma: evaluation of intensive myeloablative chemoradiation preparative regimens. J Clin Oncol 2008; 26: 5233-9.

[33] Pule MA, Savoldo B, Myers GD, et al. Virus-specific T cells engineered to coexpress tumor-specific receptors: persistence and antitumor activity in individuals with neuroblastoma. Nat Med 2008; 14: 1264-70.

[34] Porter DL, Levine BL, Kalos M, Bagg A, June CH. Chimeric Antigen Receptor Modified $\mathrm{T}$ Cells in Chronic Lymphoid Leukemia. N Engl J Med 2011; 365: 725-33.

[35] Milone MC, Fish JD, Carpenito C, et al. Chimeric receptors containing CD137 signal transduction domains mediate enhanced survival of $\mathrm{T}$ cells and increased antileukemic efficacy in vivo. Mol Ther 2009; 17: 1453-64.

[36] Chinnasamy $\mathrm{D}, \mathrm{Yu} \mathrm{Z}$, Theoret $\mathrm{M}$, et al. Gene therapy using genetically modified lymphocytes targeting VEGFR-2 inhibits the growth of vascularized syngenic tumors in mice. J Clin Investig 2010; 120: 3953-68.

[37] Moeller M, Haynes NM, Kershaw MH, et al. Adoptive transfer of gene-engineered CD4+ helper T cells induces potent primary and secondary tumor rejection. Blood 2005; 106: 2995-3003.

Received: April 18, 2013

Revised: July 02, 2013

Accepted: July 11, 2013

(C) Gu et al.; Licensee Bentham Open.

This is an open access article licensed under the terms of the Creative Commons Attribution Non-Commercial License (http://creativecommons.org/licenses/ by-nc/3.0/) which permits unrestricted, non-commercial use, distribution and reproduction in any medium, provided the work is properly cited. 